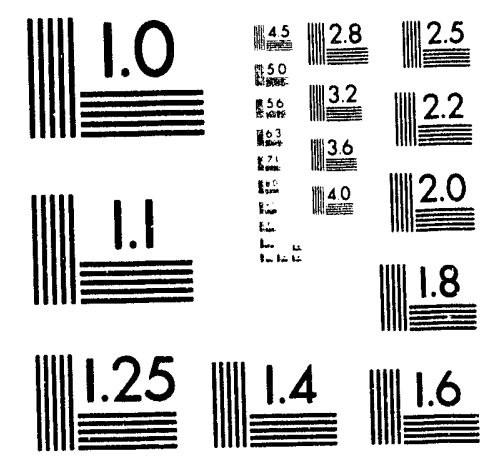



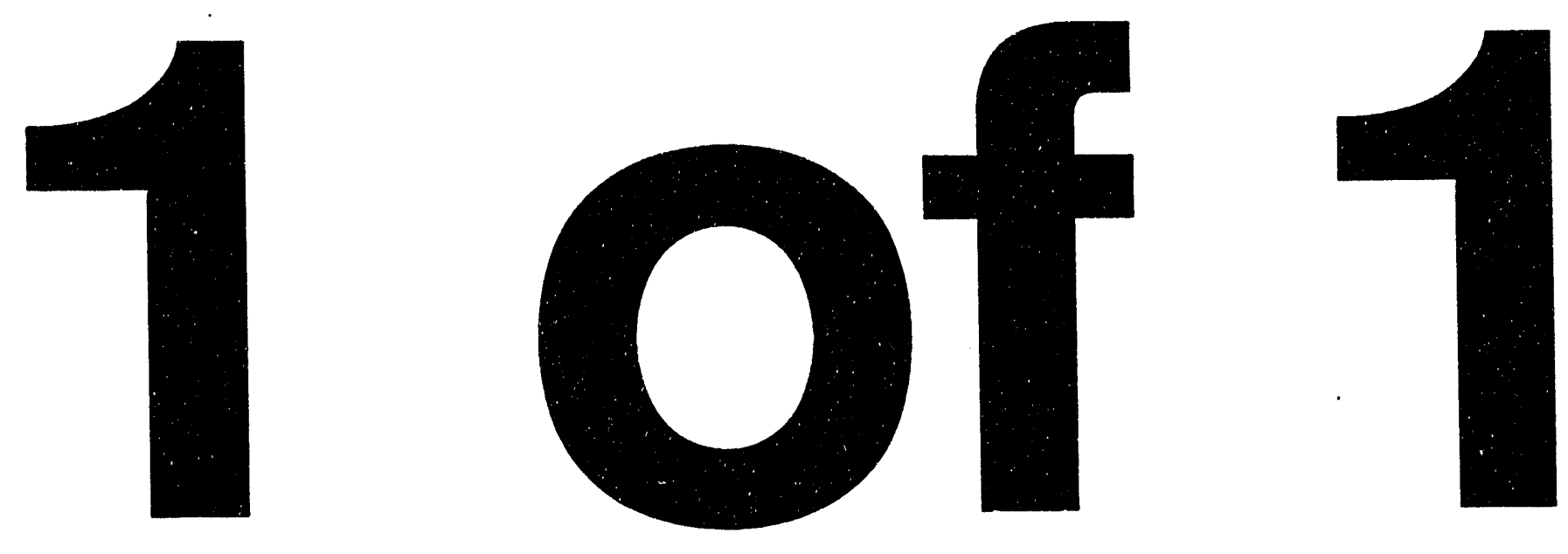
LBL-33272

\title{
Study of Coupled-Bunch Collective \\ Effects in the PEP-II B-Factory
}

\author{
J. Byrd \\ Accelerator and Fusion Research Division \\ Lawrence Berkeley Laboratory \\ University of California \\ Berkeley, California 94720
}

May 1993

This work was supported by the Director, Office of Energy Research, Office of Basic Energy Sciences, Materials Sciences Division, of the U.S. Department of Energy under Contract No. DE-AC03-76SF00098. 


\title{
Study of Coupled-bunch Collective Effects in the PEP-II B-Factory*
}

\author{
John Byrd \\ Lawrence Berkeley Laboratory \\ Berkeley, CA 94720 USA
}

\begin{abstract}
We present an overview of the calculated longitudinal and transverse coupled-bunch (CB) growth rates using the measured RF cavity higher order mode impedance and estimated resistive wall (RW) impedance for the proposed PEP-II B-Factory[1], a dual-ring electron-positron collider. We also describe a visual method of representing the effective beam impedance and corresponding growth rates which is especially useful for understanding the dependence of of growth rate on higher order mode frequency and $Q$, spread of HOM freçuencies between cells, and for determining the requirements of the $C B$ feedback systems.
\end{abstract}

\section{INTRODUCTION}

Because of the high beam current and large number of bunches at PEP-II, a proposed dual-ring electron-positron collider, CB instabilities are predicted to be one of the limiting factors in achieving the design beam intensity. Longitudinal and transverse CB feedback systems are currently being designed to combat this problem. This paper provides a detailed estimate of the growth rates in the PEP-I low energy ring (LER) using the measured HOMs of the low-power model of the PEP-II cavity and the resistive wall impedance. The gro:vth rates in the high energy ring (HER) are lower by $\sim 0.7-3$ because of the factor 3 in beam energy. This paper does not include any discussion of the $\mathrm{CB}$ effects resulting from the interaction of the beam with the fundamental mode of the cavity

\section{Coupled-Bunch IMPEDANCE}

In the case of longitudinal beam oscillations, the RF cavities provide the only sources of impedance strong enough to drive $C B$ instabilities. A series of measurements characterizing the resonant modes of the PEP-II RF cavity cell have been performed on a low-power prototype model. A summary of the properties of the monopole and dipole higher order modes (HOMs) is given in reference [2], in these proceedings.

In the case of betatron oscillations, there are two sources of impedance large enough to drive $\mathrm{CB}$ instabilities: the RF cavities and the RW impedance. An estimate of the RW impedance has been made[3], taking into account the variable resistivity, aperture, and cross section of the vacuum chamber around the ring. Because of the narrower vertical aperture in a large part of the vacuum chamber, the vertical RW impedance dominates over the horizontal.

-This work was supported by the Director, Office of Energy Research, Office of High Energy and Nuclear Physics, High Energy Physics Division, of the U.S. Department of Energy under Contract No. DE-AC03-76SF00098.
The net vertical RW impedance, scaled by the ratio of the beta function at the RW impedances and the RF cavities, is given by $Z_{\perp, R W}=0.566 \mathrm{M} \Omega / \mathrm{m} / \sqrt{f(M H z)}$.

\section{COUPLED-BUnCh GROWTH Rates}

Longitudinal Effects-The longitudinal complex frequency shift of CB mode $\ell$ for a bunched beam with $N$ equally populated and equally spaced bunches is given by[4]

$$
\begin{gathered}
\Delta \omega_{\|}^{\ell}=\frac{1}{2} \frac{I_{0} f_{r J} \alpha}{(E / e) Q_{s}} i\left[Z_{\|}\right]_{e f f}^{\ell} \\
{\left[Z_{\|}\right]_{e f \rho}^{\ell}=\sum_{p=-\infty}^{p=+\infty} \frac{\omega_{p}}{\omega_{r f}} e^{-\left(\omega_{p} \sigma_{r}\right)^{2}} Z_{\|}\left(\omega_{p}\right)}
\end{gathered}
$$

and

$$
\omega_{p}=\left(p N+\ell+Q_{s}\right) \omega_{0} .
$$

where $f_{0}$ is the revolution frequency.

The effective impedance, $\left[Z_{\|}\right]_{\text {eff }}^{\ell}$, represents the sum of the beam spectrum over the actual impedance. The complex frequency shift was evaluated using the measured parameters shown in Table 1 and the measured HOM parameters given in [2]. The imaginary part of $\mathrm{E}_{\mathrm{q}} .1$ represents a growth or damping of the CB mode and is driven by the real part of the impedance. The growth rates were calculated for the worst case of no spread in HOM frequencies among the RF cells.

Because of the sampling of the cavity wakefields by the beam, the HOM impedances are aliased into a frequency bandwidth given by $1 / 2 T_{b}$, where $T_{b}$ is the bunch separation in time. For the case of every other RF bucket filled, the frequency bandwidth is $119 \mathrm{MHz}$. We consider here the impedances aliased into the frequency band 0-119 $\mathrm{MHz}$. The summation for the effective impedance given in Eq. 2 represents this aliasing. The real part of the effective longitudinal impedance driving the longitudinal coupled bunch modes is shown graphically in Figure 1. The effer uve impedance is plotted on the left axis and the corresponding grc th rates of the unstable beam modes are plotted on the right axis. The dotted line represents the effective impedance resulting in growth for $\mathrm{CB}$ modes corresponding to upper sidebands in the frequency band 0-119 $\mathrm{MHz}$. The dashed line is the effective impedance resulting in growth for lower sidebands in the same frequency band. Note that the dashed line represents a damping impedance for the upper sideband modes in the same frequency band. Several of the aliased HOM's are labeled according to their resonant frequency as given in [2].

The CB mode freciuencies are closely spaced because of the low revolution frequency. Therefore, there is very little 


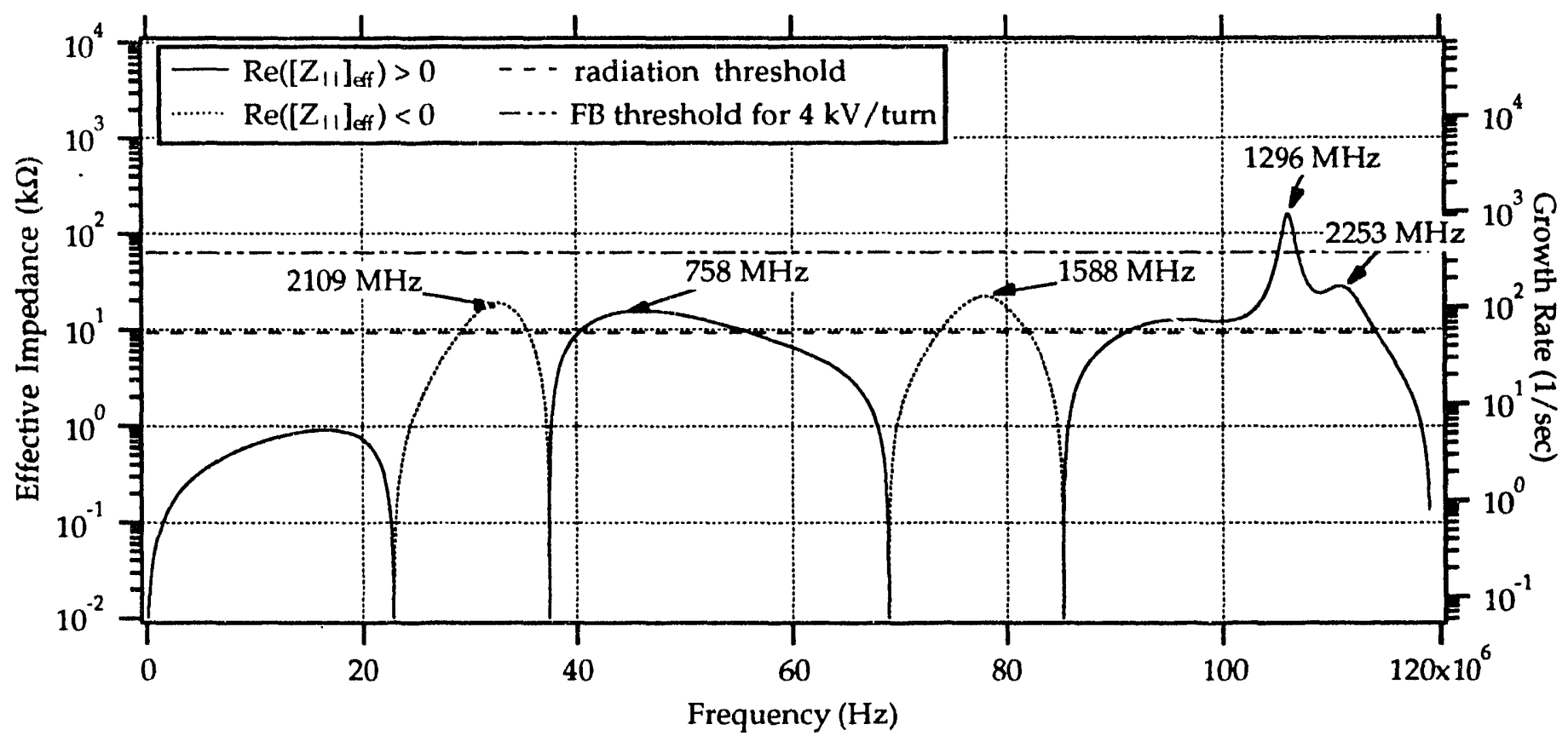

Figure 1: Longitudinal CB growth rates and the effective impedance in the 0-119 MHz frequency band. The largest $\mathrm{HOMs}$ have been labeled according to their resonant frequency in $\mathrm{MHz}$. $\mathrm{CB}$ mode frequencies are too closely spaced to be resolved on this scale.

\begin{tabular}{clc}
\hline \hline Symbol & Description & LER Value \\
\hline$E$ & Beam energy $(\mathrm{GeV})$ & 3.109 \\
$C$ & Circumference $(\mathrm{m})$ & 2199.318 \\
$f_{r f}$ & RF Freq. & $476 \mathrm{MHz}$ \\
$N$ & No. of bunches & 1746 \\
$I_{0}$ & Beam current (A) & 2.14 \\
$\alpha$ & Mom. compaction & $1.49 \mathrm{e}-3$ \\
$Q_{s}$ & Synchrotron tune & 0.05 \\
$N_{r f}$ & No. of RF cells & 10 \\
$Q_{x, y}$ & x,y $\beta$-tron tunes & $32.28,35.18$ \\
$\left\langle\beta_{x, y}\right\rangle$ & aver. $\beta_{x, y}(m)$ & $10.84,9.95$ \\
\hline \hline
\end{tabular}

Table 1: PEP-II LER parameters used for calculations.

effect of shifts in the frequencies of the HOMs because they are already heavily damped or in shifts of the $\mathrm{CB}$ mode frequencies. However, further "de-Qing" of an HOM would help by bringing down the HOM's shunt impedance even further. This could be accomplished by even more aggressive damping of the HOMs or by a spread in the HOM frequencies between the various RF cells.

Longitudinal Feedback Threshold-A CB feedback (FB) system is being designed to damp the unstable modes plotted in Figure 1. Neglecting any aid from radiation damping, the damping limit of the FB system is reached when the beam-induced voltage/turn equals the maximum $\mathrm{FB}$ kick voltage/turn. The magnitude of the beam-induced voltage/turn is given by

$$
V_{b}=I_{0} \Delta \phi Z_{\|}=I_{0} \Delta \phi_{0} Z_{\|, e s s}
$$

where $Z_{\|, e f f}(\omega)=\left(\omega / \omega_{r f}\right) Z_{\|}(\omega)$ is the where $\Delta \phi_{0}$ is the amplitude of phase modulation at the RF frequency for a $\mathrm{CB}$ mode. The maximum longitudinal kick/turn is $4 \mathrm{kV}$ and a maximum longitudinal CB amplitude of $0.03 \mathrm{rad}$ is assumed.

Transverse Effects- The transverse complex frequency shift of CB mode $\ell$ for a bunched beam with equally populated and spaced bunches is given by[4]

$$
\Delta \omega_{\perp}^{l}=-\frac{1}{2} \frac{I_{0} f_{0}}{(E / e)} i\left[\beta_{\perp} Z_{\perp}\right]_{\text {ef }}^{l}
$$

where

$$
\left[\beta_{\perp} Z_{\perp}\right]_{\text {eff }}^{\ell}=\beta_{\perp} \sum_{p=-\infty}^{p=+\infty} e^{-\left(\omega_{p} \sigma_{r}\right)^{2}} Z_{\perp}\left(\omega_{p}\right)
$$

and

$$
\omega_{p}=\left(p N+\ell+Q_{\perp}\right) \omega_{0} .
$$

$I_{0}$ is the DC current and $\beta_{\perp}$ is the transverse beta function at the location of the impedance. The complex frequency shift was evaluated using the parameters shown in Table 1 and the dipole in reference [2].

The contribution to the real part of the effective transverse impedance from the HOMs is shown graphically in Figure 2. The dotted line represents the effective impedance resulting in growth for $\mathrm{CB}$ modes corresponding to upper sidebands in the frequency band $0-119 \mathrm{MHz}$. 


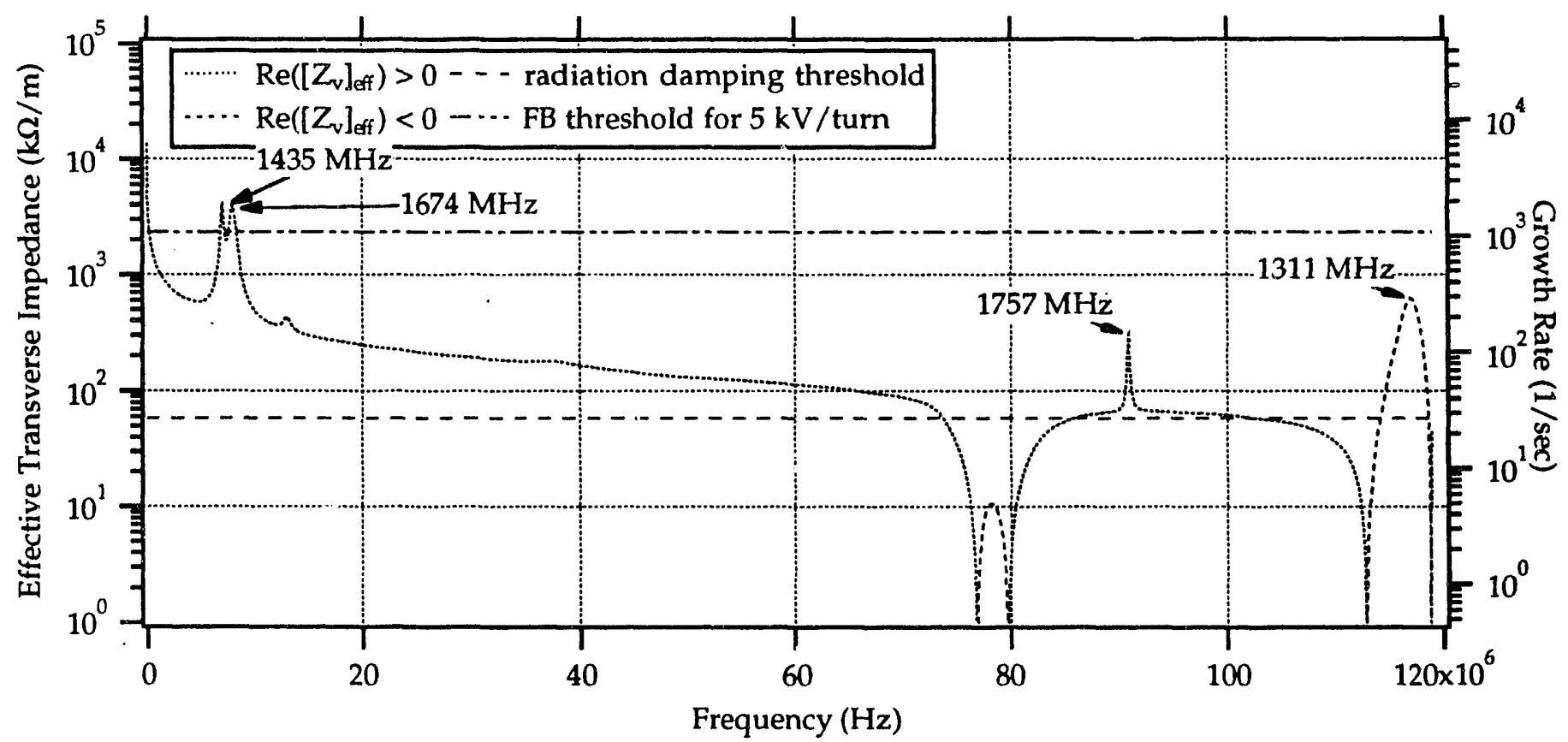

Figure 2: Vertical CB growth rates and the effective impedance in the 0-119 $\mathrm{MHz}$ frequency band from the RF cavity dipole HOM and the resistive wall impedance.

The dashed line is the effective impedance resulting in growth for lower sidebands in the same frequency band. Note that the dashed line represents a damping impedance for the upper sideband modes in the same frequency band. The radiation damping rate is also plotted.

Most of the HOMs have been well damped such that they give growth rates below the radiation damping threshold. However, there appears to be two narrowband dipole modes that can cause possible problems. Further investigation of the modes is continuing.

There is relatively little detrimental effect on the growth rates from changes in the betatron tune or changes in an HOM frequencies assuming that all RF cells change equally because of the extent of the damping of the HOMs and the relatively small revolution frequency. If the HOM frequencies in the various RF cells do not shift equally, the net HOM impedance can be reduced, thus lowering the growth rates. However, the RW impedance does not vary significantly over time. It is expected to be the dominant effect in the transverse plane.

Transverse Feedback Threshold-Neglecting any aid from radiation damping, the limit of the transverse feedback system is reached when the beam-induced voltage/turn equals the maximum feedback kick voltage/turn. The magnitude of the beam-induced voltage/turn is given by

$$
V_{b}=I_{0} \Delta x Z_{\perp}
$$

where $\Delta x$ is the transverse oscillation amplitude for a CB mode. The maximum vertical kick/turn is $5 \mathrm{kV}$ and a maximum transverse CB amplitude of $1 \mathrm{~mm}$ is assumed. As in the longitudinal case, the transverse feedback gain can be raised to sufficiently stabilize a quiescent beam.

\section{Conclusions}

Most iongitudinal CB modes are damped near or below the radiation damping rate. The rest should be damped by the FB system. One remaining monopole HOM requires further damping. The transverse plane is dominated by the RW impedance. and two other dipole HOMs which can potentially drive beam modes at a comparable race but will likely be reduced because of cell-cell differences. Landau damping from the intrabunch tune spread is not effective in damping any of the fastest growing $\mathrm{CB}$ modes at full beam current although it may help at lower currents.

The author would like to thank Glen Lambertson for many useful discussions and members of the Center for Beam Physics at LBL for their general support and encouragement.

\section{REFERENCES}

[1] Design Update for PEP-II, LBL PUB-5303 (1992).

[2] R. Rimmer, D. Goldberg, Measurement of Higher-order Mode Damping in the PEP-II Low-Yower Test Cavity, these proceedings.

[3] J. Byrd, Resistive Wall Instability at the PEP-II BFactory, CBP Tech Note-006, PEP-II AP Ncte 9-93, May 1993.

[4] M. Zisman, S. Chattopadhyay, J. Bisognano, ZAP User's manual, LBL-21270. 

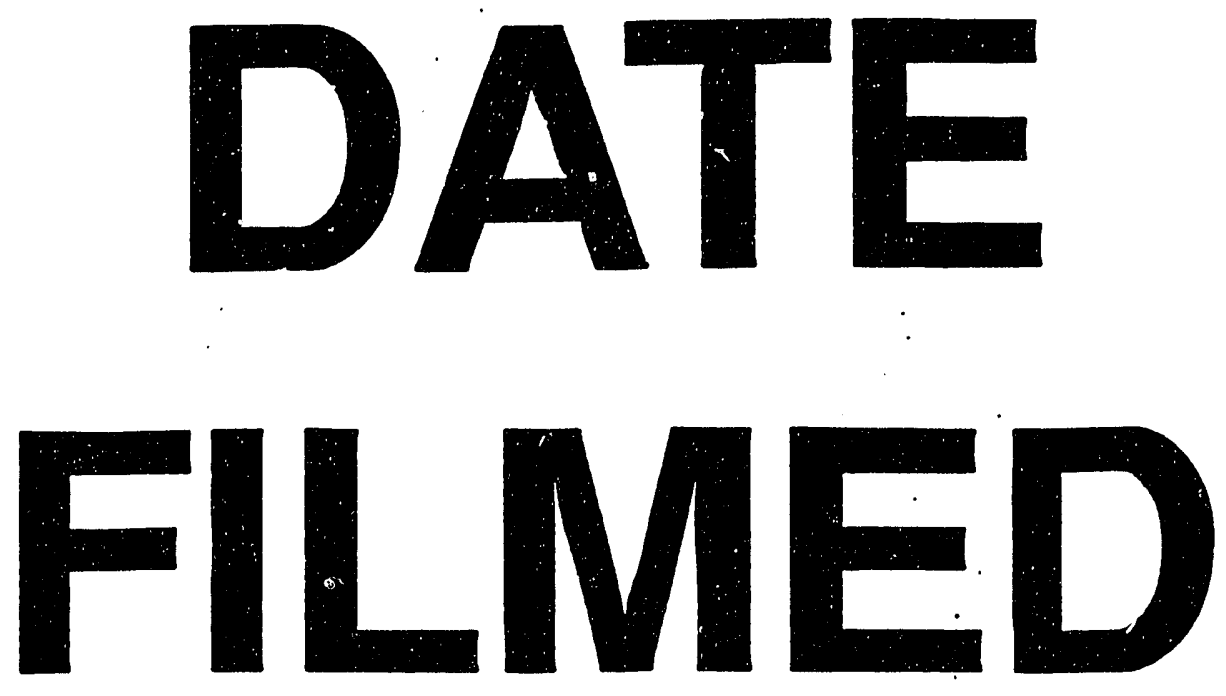

$10 / 20 / 93$
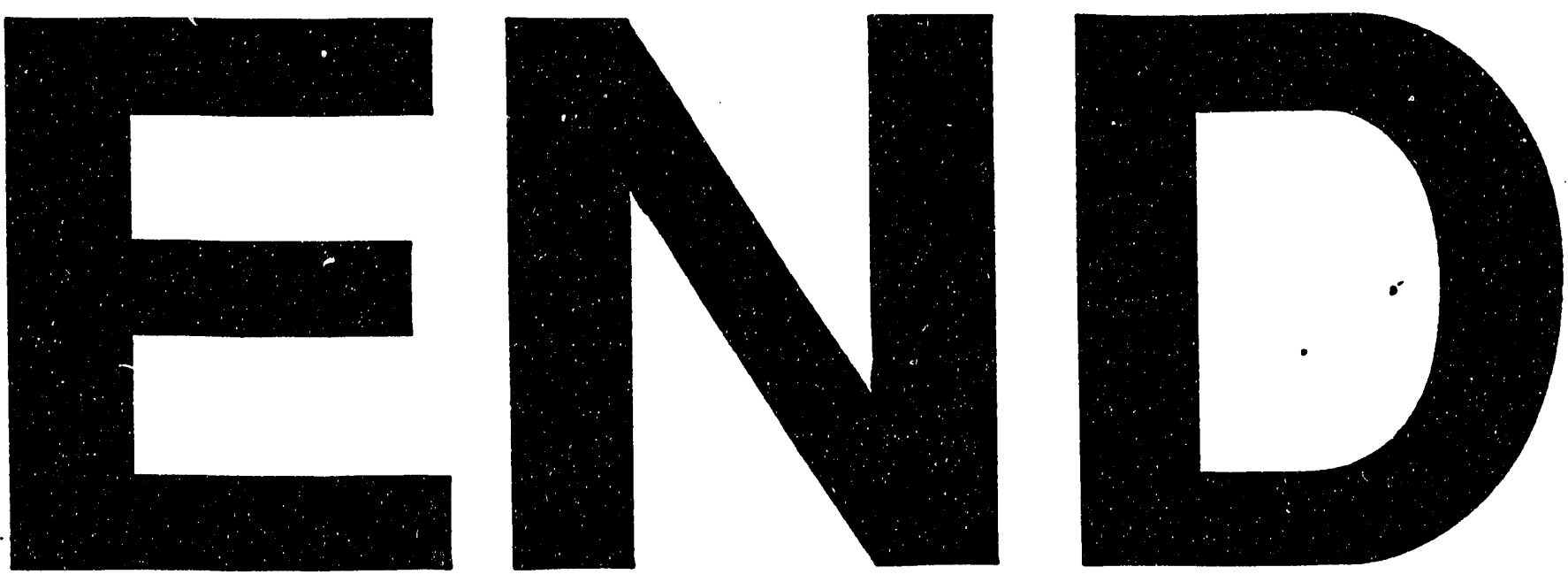
